\title{
Osteossíntese minimamente invasiva com placa para fraturas de clavícula*
}

\section{Minimally Invasive Plate Osteosynthesis for Clavicle Fractures}

\author{
Pramod Devkota1일 Balakrishnan M. Acharya ${ }^{10}$ Nabees Man Singh Pradhan ${ }^{10}$ \\ Suman Kumar Shrestha ${ }^{10}$ Abhishek Kumar Thakur ${ }^{1}{ }^{\circledR}$ Bidur Gyawali ${ }^{1 \odot}$
1 Ortopedista e traumatologista do Departamento de Ortopedia e Cirurgia do Trauma, Patan Academy of Health Sciences, Patan \\ Hospital, Lalitpur, Nepal \\ Endereço para correspondência Pramod Devkota, MS(Ortho), \\ Department of Orthopaedics and Trauma Surgery, Patan Academy of \\ Health Sciences, Patan Hospital, Lalitpur, Nepal \\ (e-mail: devkotap@gmail.com).
}

Rev Bras Ortop 2022;57(2):295-300.

\section{Resumo}

\section{Palavras-chave}

- clavícula

- fraturas ósseas

- procedimentos cirúrgicos minimamente invasivos

- placas ósseas

- fixação interna de fraturas
Objetivo Analisar o resultado radiológico, clínico e funcional das fraturas da clavícula, tratadas pela técnica de osteossíntese com placa minimamente invasiva (MIPO).

Métodos De junho de 2018 a julho de 2019, um total de 17 casos de fraturas claviculares foram tratadas com a técnica de osteossíntese com placa minimamente invasiva (MIPO), sob fluoroscopia com o braço em C. Os resultados funcionais foram avaliados por meio do escore de Constant-Murley e pelo escore de incapacidade do braço, ombro e mão (DASH). Foram analisados os resultados clínicos de consolidação, complicações, tempo cirúrgico, permanência hospitalar e infecção.

Resultados O tempo médio de acompanhamento neste estudo foi de 10,41 $\pm 1,75$ meses (variação, 8 a 14 meses). Havia 11 pacientes do sexo masculino e seis do feminino, com média de idade de 39,05 $\pm 10,76$ anos (variação de 22 a 57 anos). Todas as fraturas se consolidaram no tempo médio de 15,35 $\pm 3,08$ semanas (variação, 12 a 20 semanas). O tempo cirúrgico médio foi de $98,11 \pm 13,83$ minutos (variação, 70 a 130), sendo a permanência hospitalar média de $4,7 \pm 1,12$ dias (variação de 3 a 7 ). 0 escore de Constant-Murley médio foi de $74,82 \pm 6,36$ no $4^{\circ}$ mês e $92,35 \pm 5,48$ no $8^{\circ}$ mês do pós-operatório, o que foi estatisticamente significativo. O escore DASH médio

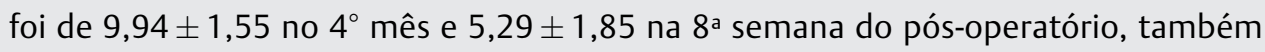
sendo estatisticamente significativo. Um paciente apresentou infecção cutânea superficial no local da incisão.

Conclusões A técnica MIPO é um método alternativo para a fixação de fraturas da clavícula, porém é tecnicamente mais desafiador, já que necessita de instalações cirúrgicas mais bem equipadas.
Estudo realizado no Departamento de Ortopedia e Cirurgia do Trauma, Patan Academy of Health Sciences, Patan Hospital, Lalitpur, Nepal. recebido

20 de Junho de 2020

aceito

01 de Dezembro de 2020

Publicado on-line

Outubro 1, 2021
DOI https://doi.org/

10.1055/s-0041-1731358.

ISSN 0102-3616.
(C) 2021. Sociedade Brasileira de Ortopedia e Traumatologia. All rights reserved.

This is an open access article published by Thieme under the terms of the Creative Commons Attribution-NonDerivative-NonCommercial-License, permitting copying and reproduction so long as the original work is given appropriate credit. Contents may not be used for commercial purposes, or adapted, remixed, transformed or built upon. (https://creativecommons.org/ licenses/by-nc-nd/4.0/)

Thieme Revinter Publicações Ltda., Rua do Matoso 170, Rio de Janeiro, RJ, CEP 20270-135, Brazil 


\author{
Abstract

\section{Keywords} \\ - clavicle \\ - fractures, bone \\ - minimally invasive \\ surgical procedures \\ - bone plates \\ - fracture fixation, \\ internal
}

Objective To analyze the radiological, clinical, and functional outcome of clavicle fractures treated by minimally invasive plate osteosynthesis (MIPO) technique.

Methods From June 2018 to July 2019, a total of 17 cases of clavicular fractures were managed using minimally invasive plate osteosynthesis (MIPO) technique under C-arm fluoroscopy. The functional outcomes were assessed using Constant-Murley score and disabilities of arm, shoulder and hand (DASH) score. Clinical results of union, complications, operative time, hospital stay and infection encountered were analyzed. Results The mean follow-up time on this study was $10.41 \pm 1.75$ months (range, 8-14 months). There were 11 male and six female patients with the mean age of the patient being $39.05 \pm 10.76$ years (range, $22-57$ years). All fractures united on the mean time of $15.35 \pm 3.08$ weeks (range, $12-20$ weeks). The mean operative time was $98.11 \pm 13.83$ minutes (range, $70-130$ ) and the mean hospital stay was $4.7 \pm 1.12$ days (range $3-7)$. The mean Constant-Murley score was $74.82 \pm 6.36$ at $4^{\text {th }}$ month and $92.35 \pm 5.48$ at $8^{\text {th }}$ month post operatively which was statistically significant. The mean DASH score was $9.94 \pm 1.55$ at $4^{\text {th }}$ month and $5.29 \pm 1.858^{\text {th }}$ week post-operatively which was statistically significant. One patient had superficial skin infection at the incision site.

Conclusions MIPO is an alternative method for the fixation of clavicle fractures but it is technically more demanding and need well equipped operating room facilities.

\section{Introdução}

As fraturas da clavícula são fraturas comuns, abrangendo de $3 \%$ a $5 \%$ de todas as fraturas, sendo que $44 \%$ das fraturas, ocorrem na região do ombro em adultos. ${ }^{1,2}$ Sessenta e cinco a 80 por cento dessas fraturas estão localizadas no terço médio, sendo que $\sim 94 \%$ são causadas por um trauma direto no ombro. ${ }^{3-5}$ No passado, o tratamento conservador das fraturas claviculares costumava ser a primeira escolha, devido ao seu bom prognóstico, em relação ao processo de recuperação e ao resultado funcional. ${ }^{6,7}$ Porém, os estudos mais recentes mostraram resultados insatisfatórios, como não consolidação da fratura, irritação do plexo braquial, queixas estéticas e função restrita do ombro, devido ao tratamento não cirúrgico das fraturas claviculares médias com desvio grave. ${ }^{8-10}$

Há uma incidência crescente de padrões de fratura da clavícula complexos, em virtude de traumatismos de alta energia e diferentes tipos de atividades esportivas. ${ }^{11,12}$ As indicações para intervenção cirúrgica nas fraturas da clavícula, conforme relatos na literatura. ${ }^{10,12}$ incluem: cominuição grave, deslocamento e encurtamento de mais de 1,5 a $2,0 \mathrm{~cm}$, ombro flutuante, pacientes politraumatizados, posicionamento subcutâneo das extremidades da fratura, levando à irritação cutânea e etc.. Atualmente, existe uma tendência ao tratamento cirúrgico das fraturas da clavícula, trazendo vantagens como o retorno precoce da função e menores taxas de consolidação incompleta e não consolidação sintomáticas. ${ }^{13,14}$ Porém, a técnica de redução aberta e a fixação interna (ORIF) das clavículas não estão isentas de complicações, sendo as mais comuns irritação da pele, devido à hardware proeminente, falha do implante, dormência, parestesia ao redor da área da cicatriz cirúrgica e infecção. ${ }^{15}$
A clavícula é um osso membranoso com irrigação vascular predominantemente periosteal, Durante a cirurgia aberta, ocorre um extenso desnudamento periosteal, podendo levar à não consolidação e infecção. ${ }^{16} \mathrm{~A}$ redução fechada e a fixação interna, podem minimizar as complicações acima mencionadas. A fixação intramedular é uma opção, mas não mantém o comprimento ou a rotação em fraturas cominutivas, sendo que a migração ou quebra da fixação pode levar à complicações sérias. ${ }^{17}$ Devido à ampla utilização da fixação biológica da fratura, levando à melhora da consolidação, a técnica MIPO vem sendo aplicada no tratamento das fraturas diafisárias da clavícula, já que atualmente existem melhores instalações cirúrgicas, tais como a eficiente disponibilidade da fluoroscopia de braço em C, assim como a mesa cirúrgica compatível com o braço em C. Tem sido relatados bons resultados clínicos e radiológicos, com a técnica MIPO na fixação da fratura clavícular. ${ }^{13,15,18-22}$

\section{Materiais e Métodos}

Foi realizado no Departamento de Ortopedia e Cirurgia do Trauma da nossa instituição, um estudo retrospectivo observacional. Este estudo foi aprovado pelo Comitê de Revisão Institucional. Desde junho de 2018 até julho de 2019, um total de 17 casos com diáfise média de fraturas claviculares (AO/OTA tipo B), foram tratados com a técnica de osteossíntese com placa minimamente invasiva (MIPO), usando a fluoroscopia de braço em C. Foram incluídos neste estudo, pacientes com mais de 18 anos de idade que apresentavam fraturas da diáfise média da clavícula, completamente deslocadas, sem nenhum contato cortical entre os fragmentos, fraturas deslocadas com encurtamento acentuado de mais de 


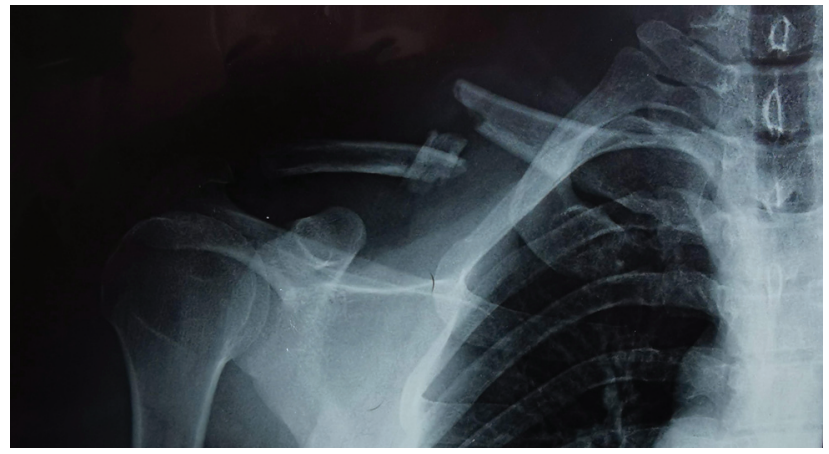

Fig. 1 Fratura cominutiva da clavícula direita de um homem de 39 anos.

1,5 a $2,0 \mathrm{~cm}$, fraturas que causam tensão cutânea e fraturas cominutivas. ( - Fig. 1) As fraturas envolvendo o terço lateral ou medial da clavícula, as fraturas expostas de clavículas, fraturas patológicas e bilaterais da clavícula, associadas a lesões neurovasculares ou lesões dos membros superiores ipsilaterais, foram excluídas deste estudo, por falta de condições financeiras para custear a placa de compressão bloqueada.

\section{Técnica Operatória}

A operação foi realizada conforme descrito no manual AO MIPO. ${ }^{23}$ Todas as cirurgias foram realizadas sob anestesia geral, com a utilização de mesa cirúrgica radiotransparente, mediante fluoroscopia de braço em C. Duas incisões na pele com cerca de 2 a $3 \mathrm{~cm}$ foram feitas nos lados medial e lateral da fratura clavícular (-Fig. 2). Foi preservado o nervo supraclavicular quando visível. A superfície superior da clavícula foi exposta. Foi criado um plano submuscular entre as duas incisões, com o uso de um elevador periosteal ou uma placa de compressão de travamento longo. A redução da fratura foi auxiliada pela manipulação do ombro e braço, sendo que a redução temporária foi mantida com o uso de fixadores externos (-Fig. 3). A placa (placa de compressão bloqueada pré-contornada para ossos clavículares) foi passada no plano submuscular. A fixação temporária foi feita com fios de Kirschner, através das luvas de broca de bloqueio,

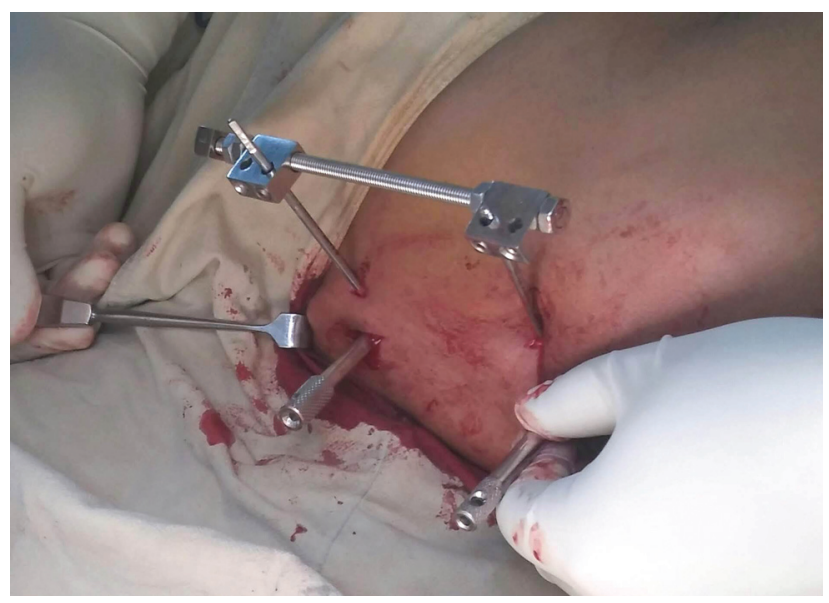

Fig. 3 A redução da fratura foi mantida com fixação externa.

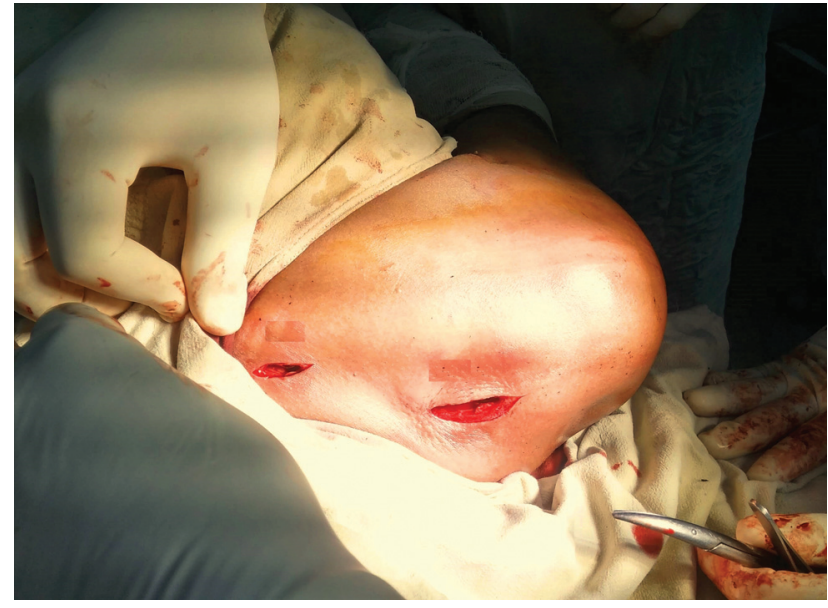

Fig. 2 Duas pequenas incisões nos lados medial e lateral da fratura da clavícula.

nos lados medial e lateral da placa. O braço em C foi utilizado para visualizar as imagens no período intra-operatório em neutro, 30 graus caudal e 30 graus cranial, a fim de confirmar a redução e a colocação das placas. A fixação definitiva foi feita com parafusos corticais de $3,5 \mathrm{~mm}$ em ambos os lados da fratura e os parafusos de cabeça bloqueada (LHS) foram usados para completar a fixação (-Fig. 4). Os ferimentos foram fechados em camadas.

Todos os pacientes foram estimulados a mover o cotovelo, punho e dedos, desde o primeiro dia do pós-operatório, sendo aconselhados a apoiar o membro superior na tipoia do braço, pelo período de seis semanas com movimentos pendulares suaves, mantendo o braço dentro da tipoia. Todas as suturas foram removidas no $14^{\circ}$ dia do pós-operatório, tendo sido descartada a tipoia na sexta semana do pósoperatório, então sendo estimulada a mobilização ativa do ombro. Durante o período de acompanhamento, foram realizadas avaliações clínicas e radiológicas, nas $6^{\underline{a}}, 12^{\underline{a}}$ e $18^{\mathrm{a}}$ semanas e nos $6^{\circ}$ e $8^{\circ}$ meses após a cirurgia. Os resultados clínicos foram avaliados usando o escore de ombro de Constant-Murley (CSS) e também o escore de incapacidade de braço, ombro e mão (DASH) ${ }^{24,25}$ A consolidação da fratura foi considerada na presença de calo ósseo em ponte no local da fratura, também foram medidas diferenças no comprimento da clavícula com lados contralaterais ilesos. O comprimento clavicular foi medido da extremidade lateral até a

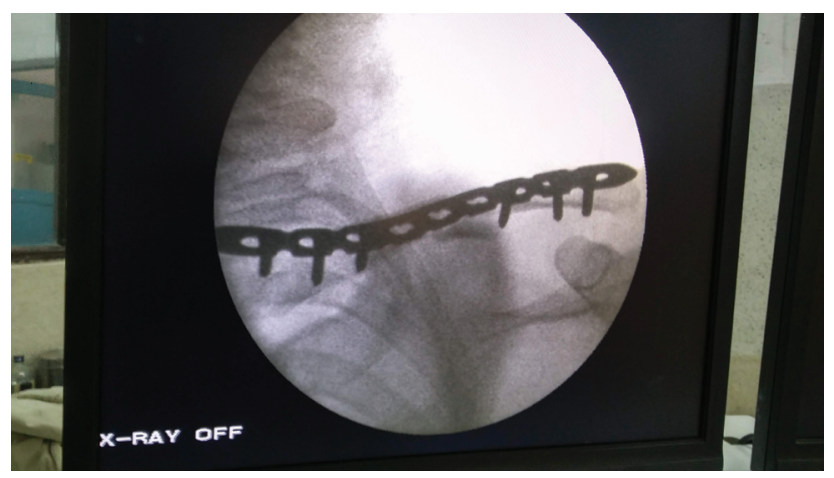

Fig. 4 Imagem intraoperatória do braço em C do mesmo paciente. 
extremidade medial da clavícula, por meio de raio-X digital (raio-X computadorizado), usando um software pré-desenhado no departamento de radiologia. Foram observadas complicações como infecção e celulite no local da cirurgia, irritação e falha do hardware, dormência, formigamento e parestesia ao redor da cicatriz cirúrgica.

\section{Análise Estatística}

A análise estatística foi realizada com o uso do software Statistical Package for the Social Sciences, versão 22 para windows, (IBM Corp., Armonk, NY, EUA). Os dados quantitativos como idade, tempo cirúrgico, internação hospitalar, tempo de acompanhamento, diferença do comprimento da clavícula e tempo de consolidação radiológica foram expressos em média \pm DP, enquanto os dados qualitativos como sexo e o lado envolvido foram expressos em porcentagem. $O$ teste $t$ de Student foi utilizado para analisar os escores DASH e ConstantMurley, entre o $4^{\circ}$ e o $8^{\circ}$ mês do pós-operatório, sendo o resultado considerado significativo, o valor de $p<0,05$.

\section{Resultados}

Houve um total de 17 casos tratados com a técnica MIPO. Havia $11(64,7 \%)$ pacientes do sexo masculino e $6(35,29 \%)$ pacientes do sexo feminino, com média de idade de 39,05 $\pm 10,76$ anos (variação de 22 a 57 anos). As causas das fraturas foram: acidentes de transito (ATR) em oito casos, queda de altura em cinco casos e agressão física em quatro casos. O tempo médio de acompanhamento neste estudo foi de 10,41 $\pm 1,75$ meses (variação, 8 a 14). Todas as fraturas foram consolidadas no tempo médio de 15,35 $\pm 3,08$ semanas (variação, 12 a 20 semanas) (-Fig. 5). O tempo cirúrgico médio foi de $98,11 \pm 13,83$ minutos (variação, 70 a 130), sendo que a permanência hospitalar média foi de $4,7 \pm 1,12$ dias (variação de 3 a 7). A pontuação média da escala Constant-Murley foi de $74,82 \pm 6,36$ (variação de 66 a 90 ) no $4^{\circ}$ mês do pós-operatório e de $92,35 \pm 5,48$ (variação de 85 a 100 ) no $8^{\circ}$ mês do pósoperatório, o que foi considerado estatisticamente significativo com p $<0,05$. A pontuação média da escala DASH foi de $9,94 \pm 1,55$ (variação de 8 a 14) no $4^{\circ}$ mês do pós-operatório e de $5,29 \pm 1,85$ (variação de 4 a 9 ) na $8^{\text {a }}$ semana do pósoperatório, o que foi estatisticamente significativo com $\mathrm{p}$ $<0,05$. A diferença média do comprimento clavicular foi de $2,23 \pm 1,39 \mathrm{~mm}$ (variação, $0-5 \mathrm{~mm}$ ), porém, foi encontrado

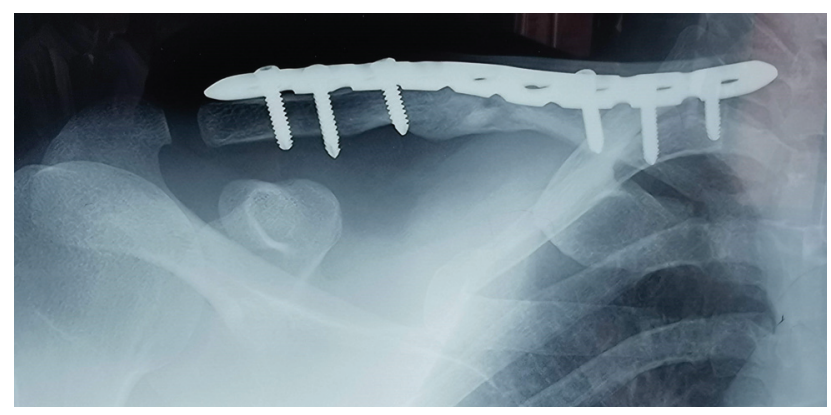

Fig. 5 Boa consolidação foi observada após 6 meses. encurtamento em nove casos do lado operado, sendo que foi encontrado alongamento em cinco casos, entretanto os escores funcionais não foram correlacionados com esses comprimentos claviculares (-Tabela $\mathbf{1}$ ).

Um paciente apresentou infecção cutânea no local da cirurgia, tendo sido tratado com antibióticos orais e curativo padrão. Não foram observados hemotórax, pneumotórax e lesões neurovasculares. Não foram registrados casos de não consolidação. Em três meses de pós-operatório, todos os pacientes obtiveram completa amplitude de movimento do ombro. Nenhum apresentou infecção profunda, nem abscesso e irritação da pele em virtude dos implantes. Não foi observada dormência ao redor do local da incisão. Todos os pacientes ficaram satisfeitos com as pequenas incisões.

\section{Discussão}

Tem havido uma tendência crescente para o tratamento cirúrgico de pacientes com fraturas clavículares desviadas agudas. ${ }^{26}$ Uma meta-análise de ensaios clínicos randomizados, mostrou que os pacientes tratados de forma não cirúrgica, apresentavam um risco maior de não consolidação ou má consolidação sintomática, do que aqueles que receberam o tratamento cirúrgico. ${ }^{27}$ Hoje em dia, a técnica MIPO tem sido amplamente utilizada no tratamento das fraturas diafisárias de ossos longos, como úmero, fêmur, tíbia e etc. ${ }^{28} \mathrm{O}$ princípio da técnica MIPO visa preservar a biologia no local da fratura, de modo que maximize o potencial de cicatrização óssea, facilitando a recuperação precoce e indolor. ${ }^{29}$ Com o intuito de lograr existo, as fraturas são geralmente reduzidas indiretamente sob a fluroscopia de braço em C. A aplicação desta técnica tem sido indicada para fraturas claviculares, com bons escores nas escalas DASH e Constant-Murley, já que apresenta menos dormência abaixo do local da incisão, restauração do comprimento da clavícula e complicações administráveis como infecção e irritação cutânea pelos implantes. ${ }^{15,18,19}$ Neste estudo, as principais causas das fraturas claviculares são os acidentes de trânsito (ATR) e as quedas de altura, respectivamente, com predominância do sexo masculino. Kundangar et al. ${ }^{15}$ e Zhang et al. ${ }^{20}$ também relataram que a maioria das fraturas foi devido à ATR,, sendo a maioria dos pacientes do sexo masculino. O tempo cirúrgico médio foi de $98,11 \pm 13,83$ minutos. Zhang et al. ${ }^{20}$ (2017) relataram tempo cirúrgico médio de 48,1 minutos, Zhang et al. ${ }^{21}$ (2016) relataram tempo médio de cirurgia de $60,2 \pm 20,1$ minutos. Nosso tempo cirúrgico foi maior do que esses dois estudos relatados, porém vem diminuindo, conforme aumenta nossa experiência com essa técnica.

A média de idade do paciente em nosso estudo foi de $39,05 \pm 10,76$ anos. Kundangar et al. $^{15}$ relataram a idade média dos pacientes como 36,1 anos, Zhang et al. ${ }^{20}$ (2017) relataram a idade média dos pacientes como 32,6 anos e Zhang et al. ${ }^{21}$ (2016) relataram a idade média dos pacientes como 48,3 anos, sendo que é comparável a este estudo. Todas as fraturas se consolidaram no tempo médio de 15,35 $\pm 3,08$ semanas, sendo semelhante ao relato de outros autores. ${ }^{15,22}$ Neste estudo, o escore médio da escala Constant-Murley foi de $74,82 \pm 6,36$ no $4^{\circ}$ mês após a cirurgia e de $92,35 \pm 5,48$ no 
Tabela 1 Demografia, resultados radiológicos e funcionais

\begin{tabular}{|c|c|c|c|c|c|c|c|c|c|c|}
\hline \multirow[t]{2}{*}{ Sexo } & \multirow[t]{2}{*}{ Idade } & \multirow{2}{*}{$\begin{array}{l}\text { Tempo de } \\
\text { consolidação } \\
\text { (semanas) }\end{array}$} & \multirow{2}{*}{$\begin{array}{l}\text { Tempo de } \\
\text { acompanhamento } \\
\text { (meses) }\end{array}$} & \multirow{2}{*}{$\begin{array}{l}\text { Tempo } \\
\text { cirúrgico } \\
\text { (minutos) }\end{array}$} & \multicolumn{2}{|c|}{ Pontuação DASH } & \multicolumn{2}{|c|}{$\begin{array}{l}\text { Pontuação } \\
\text { Constant-Murley }\end{array}$} & \multirow{2}{*}{$\begin{array}{l}\text { Estadia } \\
\text { hospitalar } \\
\text { (Dias) }\end{array}$} & \multirow{2}{*}{$\begin{array}{l}\text { Diferença do } \\
\text { Comprimento } \\
\text { Clavícular }(\mathrm{mm})\end{array}$} \\
\hline & & & & & 4 meses & 8 meses & 4 meses & 8 meses & & \\
\hline $\mathrm{M}$ & 51 & 16 & 12 & 108 & 14 & 9 & 70 & 90 & 5 & $2^{*}$ \\
\hline$M$ & 38 & 12 & 10 & 110 & 10 & 7 & 68 & 88 & 4 & $4^{*}$ \\
\hline$M$ & 55 & 20 & 9 & 105 & 10 & 5 & 70 & 85 & 5 & $3^{*}$ \\
\hline $\mathrm{F}$ & 27 & 12 & 11 & 90 & 12 & 4 & 80 & 98 & 7 & $1^{*}$ \\
\hline$M$ & 22 & 16 & 9 & 70 & 10 & 4 & 78 & 95 & 4 & 2 \\
\hline $\mathrm{M}$ & 39 & 16 & 8 & 115 & 8 & 5 & 90 & 100 & 4 & 0 \\
\hline $\mathrm{F}$ & 25 & 20 & 13 & 90 & 9 & 4 & 80 & 92 & 5 & 5 \\
\hline$M$ & 49 & 16 & 11 & 105 & 10 & 5 & 70 & 86 & 4 & 0 \\
\hline $\mathrm{F}$ & 32 & 12 & 8 & 130 & 9 & 6 & 80 & 98 & 3 & 4 \\
\hline $\mathrm{M}$ & 38 & 20 & 9 & 90 & 8 & 4 & 80 & 95 & 5 & 0 \\
\hline $\mathrm{M}$ & 33 & 12 & 8 & 95 & 9 & 5 & 66 & 88 & 3 & 3 \\
\hline $\mathrm{F}$ & 57 & 20 & 11 & 80 & 10 & 6 & 70 & 85 & 6 & 2 \\
\hline $\mathrm{F}$ & 28 & 16 & 14 & 90 & 9 & 4 & 70 & 85 & 5 & 2 \\
\hline$M$ & 38 & 16 & 10 & 100 & 8 & 6 & 80 & 100 & 7 & 3 \\
\hline$M$ & 32 & 12 & 12 & 105 & 12 & 7 & 70 & 90 & 4 & 2 \\
\hline$M$ & 47 & 13 & 10 & 85 & 10 & 4 & 70 & 90 & 5 & $2^{*}$ \\
\hline$M$ & 53 & 12 & 12 & 100 & 11 & 5 & 80 & 100 & 4 & 3 \\
\hline
\end{tabular}

*Lado alongado.

$8^{\circ}$ mês após a cirurgia. O escore médio da escala DASH foi de $9,94 \pm 1,55$ no $4^{\circ}$ mês após a cirurgia e de $5,29 \pm 1,85$ no $8^{\circ}$ mês após a cirurgia. Outros autores também usaram essas ferramentas de pontuação, a fim de avaliar a recuperação funcional dos pacientes tratados, após o emprego da técnica MIPO no tratamento cirúrgico das fraturas clavículares. ${ }^{15,20-22}$ Kundangar et al. ${ }^{15}$ relataram o escore médio da escala Constant-Murley de 92,95 $\pm 5,83$ e o escore DASH de $4,63 \pm 3,23$, Zhang et al. ${ }^{21}$ encontraram escore na escala de Constant-Murley de $99 \pm 1,8$ e escore DASH de 3,8 $\pm 2,9$. Essas pontuações funcionais relatadas são comparáveis aos nossos resultados. Percebemos a progressão desses escores, com resultados estatisticamente significativos no tempo final de acompanhamento ( $8^{\circ}$ mês após a cirurgia) que se deve à consolidação da fratura e a fisioterapia.

Outros autores relataram boa consolidação das fraturas e no escore da escala DASH, para as fraturas clavículares tratadas com a técnica ORIF. ${ }^{2,30}$ Kundangar et al. ${ }^{31}$ fizeram um estudo comparativo entre as técnicas ORIF e MIPO, onde foi obtido melhores escores no grupo ORIF, embora não tenham sido estatisticamente significativos, porém o grupo MIPO apresentou menos dormência abaixo da cicatriz. Beire et al. ${ }^{32}$ relataram que a técnica MIPO reduziu significativamente a dormência da parede torácica anterior, assim como, apresentou menos dor no pós-operatório, em comparação com a técnica ORIF, porém sem diferença estatisticamente significativa.

A diferença média do comprimento clavicular foi de $2,2 \pm 1,52 \mathrm{~mm}$. Kundangar et al. ${ }^{31}$ relataram menor diferença no comprimento clavicular no grupo cirúrgico aberto, do que no grupo MIPO, com resultado estatisticamente significativo. O tempo médio de internação foi de $4,7 \pm 1,12$ dias. Wang et al. $^{33}$ relataram uma internação hospitalar média de 6,5 dias, sendo comparável aos nossos resultados. Um paciente apresentou infecção cutânea no local da incisão, sendo tratada com curativo padrão e antibióticos orais. Não encontramos nenhuma infecção profunda, como abscesso nos locais operatórios e irritação da pele pelos implantes. Outros estudos relataram um pequeno número de pacientes com irritação de hardware e infecções irrelevantes. ${ }^{15,31}$ Não foram observados hemotórax, pneumotórax e lesões neurovasculares. Kim et al. ${ }^{34}$ relataram um pneumotórax, após a utilização da técnica MIPO em fratura da clavícula. Não foi observado nenhum caso de não consolidação, Outros autores com as técnicas de MIPO e ORIF também não relataram não consolidação. ${ }^{2,22,30-33}$ Nos três meses do pós-operatório, todos os pacientes obtiveram completa amplitude do movimento do ombro, não tendo sido observada dormência ao redor do local da incisão. A maioria dos outros autores também relatou resultados semelhantes ${ }^{15,20-22}$

\section{Limitações}

As deficiências do nosso estudo foram o tamanho da amostra, relativamente pequena, além do curto período de tempo de acompanhamento. Este estudo não foi comparativo, nem avaliou os resultados clínicos com o lado contralateral saudável.

\section{Conclusão}

Este estudo demonstrou que a técnica MIPO pode ser aplicada às fraturas da diáfise clavicular, com resultados clínicos e radiológicos satisfatórios, em termos de consolidação óssea, restauração do comprimento da clavícula e recuperação do movimento do ombro. Podendo ser uma alternativa ao método ORIF convencional. 


\section{Suporte Financeiro}

Não houve suporte financeiro de fontes públicas, comerciais, ou sem fins lucrativos.

\section{Conflito de Interesses}

Todos os autores declaram não haver conflito de interesses.

\section{Agradecimento}

Dr. Toya Raj Bhatta, Dr. Amrit Shrestha, Dr. Prabhav Pokhrel pelo apoio durante este estudo.

\section{Contribuições dos Autores}

Todos os autores contribuíram com a concepção e o desenho do estudo. A preparação do material, a coleta e a análise dos dados foram realizadas por P. Devkota, B.M. Acharya e N.M.S Pradhan. O primeiro rascunho do manuscrito foi escrito por P. Devkota, sendo que todos os autores comentaram as versões anteriores do manuscrito. Todos os autores leram e aprovaram o manuscrito final.

\section{Referências}

1 Khan LA, Bradnock TJ, Scott C, Robinson CM. Fractures of the clavicle. J Bone Joint Surg Am 2009;91(02):447-460

2 Hehn FHS, Bonavides PSG, Oliveira Júnior AN, Silva HCG, Back Neto M, Stipp WN. Clinical Evaluation of the Surgical Treatment of Midshaft Clavicle Fractures at a Hospital in the South of Santa Catarina. Rev Bras Ortop (Sao Paulo) 2020;55(01):100-105

3 Nordqvist A, Petersson C. The incidence of fractures of the clavicle. Clin Orthop Relat Res 1994;(300):127-132

4 Rowe CR. An atlas of anatomy and treatment of midclavicular fractures. Clin Orthop Relat Res 1968;58(58):29-42

5 Stanley D, Trowbridge EA, Norris SH. The mechanism of clavicular fracture. A clinical and biomechanical analysis. J Bone Joint Surg Br 1988;70(03):461-464

6 Eskola A, Vainionpää S, Myllynen P, Pätiälä H, Rokkanen P. Outcome of clavicular fracture in 89 patients. Arch Orthop Trauma Surg 1986;105(06):337-338

7 Andersen K, Jensen PO, Lauritzen J. Treatment of clavicular fractures. Figure-of-eight bandage versus a simple sling. Acta Orthop Scand 1987;58(01):71-74

8 Stanley D, Norris SH. Recovery following fractures of the clavicle treated conservatively. Injury 1988;19(03):162-164

9 Robinson CM, Court-Brown CM, McQueen MM, Wakefield AE. Estimating the risk of nonunion following nonoperative treatment of a clavicular fracture. J Bone Joint Surg Am 2004;86(07):1359-1365

10 Hill JM, McGuire MH, Crosby LA. Closed treatment of displaced middle-third fractures of the clavicle gives poor results. J Bone Joint Surg Br 1997;79(04):537-539

11 Postacchini F, Gumina S, De Santis P, Albo F. Epidemiology of clavicle fractures. J Shoulder Elbow Surg 2002;11(05):452-456

12 Souza NASM, Belangero PS, Figueiredo EA, Pochini AC, Andreoli CV, Ejnisman B. Displaced midshaft clavicle fracture in athletes should we operate? Rev Bras Ortop 2018;53(02):171-175

13 Jiang H, Qu W. Operative treatment of clavicle midshaft fractures using a locking compression plate: comparison between miniinvasive plate osteosynthesis (MIPPO) technique and conventional open reduction. Orthop Traumatol Surg Res 2012;98(06): 666-671

14 Robinson CM, Goudie EB, Murray IR, et al. Open reduction and plate fixation versus nonoperative treatment for displaced midshaft clavicular fractures: a multicenter, randomized, controlled trial. J Bone Joint Surg Am 2013;95(17):1576-1584
15 Kundangar RS, Mohanty SP, Bhat NS. Minimally invasive plate osteosynthesis (MIPO) in AO/OTA type B displaced clavicle fractures. Musculoskelet Surg 2019;103(02):191-197

16 Zenni EJ Jr, Krieg JK, Rosen MJ. Open reduction and internal fixation of clavicular fractures. J Bone Joint Surg Am 1981;63 (01):147-151

17 Millett PJ, Hurst JM, Horan MP, Hawkins RJ. Complications of clavicle fractures treated with intramedullary fixation. J Shoulder Elbow Surg 2011;20(01):86-91

18 Jung GH, Park CM, Kim JD. Biologic fixation through bridge plating for comminuted shaft fracture of the clavicle: technical aspects and prospective clinical experience with a minimum of 12-month follow-up. Clin Orthop Surg 2013;5(04):327-333

19 Lee HJ, Oh CW, Oh JK, et al. Percutaneous plating for comminuted midshaft fractures of the clavicle: a surgical technique to aid the reduction with nail assistance. Injury 2013;44(04):465-470

20 Zhang T, Chen W, Sun J, Zhang Q, Zhang Y. Minimally invasive plate osteosynthesis technique for displaced midshaft clavicular fracture using the clavicle reductor. Int Orthop 2017;41(08):1679-1683

21 Zhang Y, Xu J, Zhang C, Sun Y. Minimally invasive plate osteosynthesis for midshaft clavicular fractures using superior anatomic plating. J Shoulder Elbow Surg 2016;25(01):e7-e12

22 Sohn HS, Shon MS, Lee KH, Song SJ. Clinical comparison of two different plating methods in minimally invasive plate osteosynthesis for clavicular midshaft fractures: A randomized controlled trial. Injury 2015;46(11):2230-2238

23 Phiphobmongkol V, Sommer C. Cases - Clavicle. In: Bast R Bavonratanvavech S, Pesantez R. AOTrauma - Minimally Invasive Plate Osteosynthesis. 2nd ed. New York: Thieme; 2012:153-178

24 Constant CR, Murley AH. A clinical method of functional assessment of the shoulder. Clin Orthop Relat Res 1987; (214):160-164

25 Hudak PL, Amadio PC, Bombardier CThe Upper Extremity Collaborative Group (UECG) Development of an upper extremity outcome measure: the DASH (disabilities of the arm, shoulder and hand) [corrected]. [corrected]Am J Ind Med 1996;29(06): 602-608

26 Canadian Orthopaedic Trauma Society. Nonoperative treatment compared with plate fixation of displaced midshaft clavicular fractures. A multicenter, randomized clinical trial. J Bone Joint Surg Am 2007;89(01):1-10

27 McKee RC, Whelan DB, Schemitsch EH, McKee MD. Operative versus nonoperative care of displaced midshaft clavicular fractures: a meta-analysis of randomized clinical trials. J Bone Joint Surg Am 2012;94(08):675-684

28 Perren SM. The technology of minimally invasive percutaneous osteosynthesis (MIPO). Injury 2002;33(Suppl 1):VI-VII

29 Shin SJ, Sohn HS, Do NH. Minimally invasive plate osteosynthesis of humeral shaft fractures: a technique to aid fracture reduction and minimize complications. J Orthop Trauma 2012;26(10): 585-589

30 Ibrahim S, Meleppuram JJ. Retrospective study of superior anterior plate as a treatment for unstable (Neer type 2) distal clavicle fractures. Rev Bras Ortop 2017;53(03):306-313

31 Beirer M, Postl L, Crönlein M, et al. Does a minimal invasive approach reduce anterior chest wall numbness and postoperative pain in plate fixation of clavicle fractures? BMC Musculoskelet Disord 2015;16:128

32 Kundangar R, Singh KA, Mohanty SP, Eshwari K. Clinical outcome of internal fixation of middle third clavicle fractures using locking compression plate: Comparison between open plating and MIPO.J Orthop 2019;16(05):414-418

33 Wang X, Wang Z, Xia S, Fu B. Minimally invasive in the treatment of clavicle middle part fractures with locking reconstruction plate. Int J Surg 2014;12(07):654-658

34 Kim MK, Lee HJ, You AH, Kang HYP. Pneumothorax after minimally invasive plate osteosynthesis for midshaft clavicle fracture: A case report. Medicine (Baltimore) 2019;98(33):e16836 